\title{
IDEAS Y FINES DE LA UNIVERSIDAD*
}

\author{
José Joaquín Brunner \\ Universidad Diego Portales
}

\begin{abstract}
RESUMEN: La historia de la universidad ha sido acompañada por un discurso sobre su razón de ser, su razón de existir. Este ensayo arguye que retazos de aquellas justificaciones se utilizan para dar sustento al debate actual sobre el futuro de la universidad en Chile, sin hacerse cargo de que la realidad de la educación superior hoy es muy distinta a la realidad en que aquellos discursos originales nacieron.

Palabras Clave: universidad, idea de universidad, postmodernismo, paradigmas, sociedad de masas.
\end{abstract}

\section{THE CONCEPT AND PURPOSE OF UNIVERSITIES}

ABSTRACT: The history of universities has been accompanied by discourse on their raison d'etre, their reason for being. This essay argues that remnants of those rationales are used to support the current debate on the future of universities in Chile, but without addressing the fact that higher education today is quite different from what it was when the original discourse began.

KEYwORDs: university, the concept of universities, post-modernism, paradigms, mass society.

José Johquín Brunner. PhD en sociología por la Universidad de Leiden, Países Bajos. Profesor titular de la Universidad Diego Portales, donde dirige la cátedra Unesco de Estudios Comparados de Educación Superior y el Programa de Doctorado en Sistemas, Políticas y Gestión de la Educación Superior ofrecido conjuntamente por las universidades Diego Portales y de Leiden. Miembro de número de la Academia de Ciencias Sociales, Políticas y Morales del Instituto de Chile. Email: josejoaquin.brunner@cpce.com.

* Versión revisada del texto presentado en la Academia de Ciencias Sociales, Políticas y Morales del Instituto de Chile, el 27 de abril de 2015. 
M i propósito es explorar el campo de ideas, discursos y narrativas en torno a la universidad y a las políticas de educación superior. En particular, me interesa la relación entre discursos sobre la "idea de la universidad" y narrativas referidas a los sistemas contemporáneos de educación superior y a su reforma en el caso de Chile.

I.

Desde Kant en adelante la universidad moderna ha vivido rodeada de un discurso de ideas y fines. Ha sido pensada y se ha representado como la institucionalización de una "idea". El mismo Kant sugirió que la universidad, como toda institución artificial, tenía por fundamento una idea racional. ${ }^{1}$ No muy distinto pensaba nuestro filósofo Jorge Millas, para quien la misión social de la universidad consistía en "ser el locus veritatis, el locus rationis de la comunidad nacional". ${ }^{2}$

Pues bien, si con un salto de la imaginación nos situamos ahora en el presente, ¿se mantiene esa visión y ese fundamento racional hasta hoy?

La respuesta del filósofo francés Jacques Derrida, en su conferencia frecuentemente citada de la Universidad de Cornell, del año 1983, resuena con igual fuerza que en el origen moderno de esta institución.

“Tener una 'razón de ser' — decía él en esa ocasión- es tener una justificación para existir, tener un sentido, una finalidad, una destinación. Es asimismo tener una causa, dejarse explicar, según el 'principio de razón', por una razón que es también una causa (ground, Grund), es decir también un fundamento y una fundación". 3

Y a continuación declaraba: "Que yo sepa, jamás se ha fundado un proyecto de universidad contra la razón. Se puede, por consiguiente, pensar razonablemente que la razón de ser de la universidad siempre

${ }^{1}$ Immanuel Kant, La contienda entre las facultades de filosofía y teología (Madrid: Editorial Trotta, 1999), 5.

${ }^{2}$ Jorge Millas, Idea y defensa de la universidad (Santiago: Ediciones Universidad Diego Portales, 2012), 80.

3 Jacques Derrida, "Las pupilas de la universidad. El principio de razón y la idea de la universidad", traducción de Cristina de Peretti, en Cómo no hablar y otros textos (Barcelona: Proyecto A, 1997), 1, disponible en https://cv2.sim.ucm. es/moodle/file.php/11491/OtrosTextosFilosofYPolitica/Derrida-Las_pupilas_de_la_ Universidad.pdf/. 
fue la razón misma, así como una cierta relación especial de la razón con el ser". ${ }^{4}$

A lo largo de los últimos doscientos años, entonces, la "idea" y los fines de la universidad han sido comunicados por medio de una serie de discursos que hablan de razón, verdad y conciencia; de ideales de formación humana; de bien público y de vocación de servicio respecto de los valores honrados por una u otra comunidad nacional. Las metáforas rectoras de esos discursos nos servirán para recapitular esa trayectoria de "ideas" y misiones de la universidad; si no de su ser, a lo menos de su deber ser imaginado a lo largo del curso de la modernidad.

En paralelo con la línea que de Kant a Derrida identifica universidad con razón, una segunda línea de argumentación se pregunta qué formar: ¿un "hombre cultivado" de tipo gentleman o bien un profesional experto, según la clásica distinción de Max Weber? El Cardenal Newman, en su obra The Idea of a University, es probablemente el mayor exponente del ideal de la formación del gentleman, a través de una educación liberal o en artes liberales, en la que los conocimientos adquiridos valen por sí mismos, sin tener a la vista un uso o utilidad posteriores, al tiempo que la persona cultiva un carácter apropiado al estatus social que está llamada a ocupar. ${ }^{5}$ Hasta hoy hay colleges y universidades - en Inglaterra y los Estados Unidos especialmente- que mantienen en alto este ideal.

En el extremo opuesto - el de la formación especializada del profesional - opera como modelo el programa napoleónico, con su énfasis en la provisión estatal, un currículo centralmente regulado, exámenes oficiales y escuelas profesionales separadas de las facultades de artes y letras. Este tipo de formación alcanza su expresión más alta en las prestigiosas grandes écoles que preparan a las élites francesas para la administración del Estado, los negocios y la academia. ${ }^{6}$

\footnotetext{
${ }^{4}$ Ibídem, 4.

${ }^{5}$ John Henry Newman, The Idea of a University, editado por Frank M. Turner (New Haven, CT: Yale University Press, 1996), 76-91.

${ }^{6}$ Véase Walter Rüegg, ed., "Themes", en A History of the University in Europe. Volumen III. Universities in the Nineteenth and Early Twentieth Centuries (1800-1945) (New York: Cambridge University Press, 2004), 3-31. Asimismo, Christophe Charle, "Patterns", en la misma obra, 33-80.
} 
Al medio, por así decir, de la formación de gentlemen y expertos, el idealismo alemán — con Wilhelm von Humboldt y Friedrich Schleiermacher a la cabeza - crea en Prusia un tercer modelo peculiar. La universidad alemana (partiendo por Berlín) se propone ofrecer una educación académica fundada en principios filosóficos — como unidad entre ciencias y humanidades y de investigación y docencia-, en un ambiente donde maestros y estudiantes trabajan juntos en seminarios, y los jóvenes aprenden a desarrollar sus habilidades de investigar, hacer descubrimientos y presentarlos, según Schleiermacher. ${ }^{7}$ A cambio de protección, autonomía y financiamiento otorgados por el Estado, la nueva universidad alemana asegura a sus miembros la posibilidad de trabajar en Einsamkeit und Freiheit, aislamiento y libertad, recluidos en una "torre de marfil" al precio de no intervenir en las controversias del día. Los jóvenes a su vez pueden aspirar allí a una auténtica Bildung, esto es, "al más alto y armónico (auto)desarrollo o (auto)cultivo de las facultades humanas hasta alcanzar una plena y consistente forma" como persona moral y buen ciudadano, idea enunciada por Wilhelm von Humboldt. $^{8}$

Dentro de esta misma línea argumental hay todavía otra propuesta, la de Ortega y Gasset de los años 1930, según la cual la misión de la universidad en una sociedad de masas es educar al hombre medio, formándolo para el desempeño de profesiones especializadas al mismo tiempo que como un hombre culto, familiarizado con los conocimientos esenciales de física, biología, historia, sociología y filosofía. ${ }^{9}$

Una tercera línea de argumentación busca definir a la universidad por su función en la sociedad. Karl Jaspers, el filósofo existencialista alemán, la preside con su famoso dictum: "La universidad es la sede en la cual la sociedad y el Estado permiten el florecimiento de la más clara conciencia de la época". ${ }^{10}$ Una universidad, por tanto, que actúa - con-

${ }^{7}$ Citado en Charle, "Patterns", 48.

${ }^{8}$ Citado en Henrik Bohlin, "Bildung and Moral Self-Cultivation in Higher Education: What Does it Mean and How Can it be Achieved?", Forum on Public Policy, Södertörns högskola, Suecia, 2008, 1.

9 José Ortega y Gasset, Misión de la universidad (Madrid: Revista de Occidente - Alianza Editorial, 2007), 53-79.

${ }^{10}$ Karl Jaspers, "La idea de la universidad", traducido por Agustina Schroeder Castelli, en La idea de la universidad alemana, de varios autores (Buenos Aires: Editorial Sudamericana, 1959), 342. 
forme a su propia autonomía, libertad de sus miembros y razón de sercomo un órgano reflexivo de la sociedad; que asume la responsabilidad de ser conciencia intelectual de su tiempo, dice Jaspers en otra parte. ${ }^{11}$

Durante los años 1960, sectores intelectuales latinoamericanos hicieron propia esa "idea" de universidad como conciencia, transformándola, bajo el efecto combinado del pensamiento católico progresista y del marxismo, en conciencia de la nación, el pueblo o una clase social. Así, el famoso Documento de Buga, preparado para el Celam en 1967, definía a la universidad como "conciencia viva de la comunidad humana a la cual pertenece", compromiso que debía llevarla a apartarse de la "torre de marfil" prusiana para volverse, como decía el mismo documento, un "foco de concientización de la realidad histórica". ${ }^{12}$ El riesgo, claro está, como anticipó tempranamente José Medina Echeverría, uno de los padres de la sociología iberoamericana, era comprometer a la universidad militantemente con los "ruidos de la calle", dejándola a merced de las turbulencias del entorno. Efectivamente, la metáfora de la universidad-conciencia experimentó una progresiva radicalización en nuestra región; léase: conciencia moral y política, conciencia crítica, conciencia de cambio, conciencia revolucionaria y antiimperialista.

Dentro de esta misma línea de argumentación funcional es necesario mencionar, por último, a Clark Kerr, presidente de la Universidad de California en Berkeley, cuyo libro del año 1964 - de título impecablemente funcionalista: The Uses of the University - acuño el término multiversidad, que el autor asimilaba a "una ciudad de infinita variedad"; una serie de comunidades sueltamente acopladas bajo un nombre común, una junta de gobierno y unos propósitos relacionados entre sí. Nace así un nuevo tipo de institución, decía el autor, ni pública ni privada, no enteramente del mundo ni aparte de él, una estación de servicio para todo público; en suma, una institución surgida de un imperativo histórico antes que de una elección racional entre alternativas igualmente elegantes. ${ }^{13}$

${ }^{11}$ Karl Jaspers, The Idea of the University, editado por Karl W. Deutsch (Boston: Beacon Press, 1959), 121.

12 Consejo Episcopal Latinoamericano (Celam), "Los cristianos en la universidad”, Departamento de Educación y de Pastoral Universitaria, Bogotá, 1979, 18 y 23.

${ }^{13}$ Clark Kerr, The Uses of the University (New York: Harper Torchbooks, 1963), 1-9. 
Con la multiversidad hemos arribado al presente; un territorio posmoderno, donde la escena mundial se halla poblada por miles, decenas de miles, quizá cien mil instituciones de educación superior. Más de diez mil existen sólo en América Latina, de las cuales 3.500 ostentan el nombre de universidad. No hay ninguna "idea" que las funde, justifique o pueda incluirlas a todas. Resulta imposible predicar que cada una individualmente sea un locus veritatis o que colectivamente tengan la misma razón de ser o institucionalicen por igual la conciencia de la nación o formen coherentemente un mismo tipo humano. ${ }^{14}$

Incluso más: a esta altura podemos preguntarnos, igual como hace un historiador británico, por qué la universidad moderna necesitaría una "idea" fundante, en el sentido de una razón de ser o una justificación. Confiesa que le parece una cuestión misteriosa. ${ }^{15}$ Lo mismo me sucede a mí.

Digamos así: no es a Kant, Jaspers o Derrida, sino que a Max Weber y a los estudiosos de las organizaciones académicas a quienes hoy necesitamos recurrir. En efecto, debemos examinar si acaso la premisa misma de que la vasta y extraordinariamente compleja estructura del moderno sistema universitario está sostenida por una "idea" no resulta en extremo irreal. Seguramente arribaríamos a la conclusión de que "las organizaciones ya no materializan una idea", como dice Habermas en este punto, refutando el idealismo alemán. ${ }^{16}$

En efecto, el estudio de las organizaciones académicas no puede soslayar el hecho de la diferenciación y especialización funcional de los sistemas de educación superior, ni la variedad de tribus y subculturas disciplinarias que a partir de allí se forman, cada una con sus propios referentes cognitivos y normativos. No hay cabida ya para una concepción unitaria de las ciencias a la que todavía aspiraba Humboldt ni para

14 Ver José Joaquín Brunner, "Universidad para todos", Estudios Públicos 124 (2011): 151-170, disponible en http://www.cepchile.cl/1_4980/doc/universidad_para_todos.html/.

15 Sheldon Rothblatt, The Modern University and Its Discontents. The Fate of Newman's Legacies in Britain and America (Cambridge: Cambridge University Press, 1997), cap. 1.

16 Jürgen Habermas y John R. Blazek, "The Idea of the University: Learning Processes", New German Critique 41 (Special Issue on the Critiques of the Enlightenment, 1987): 3-22. 
una jerarquía de las facultades como defendía Kant. La "idea de la universidad" ha dado paso al bazar cultural de la posmodernidad.

Pero, ¿qué es la posmodernidad? Aquí será entendida como una transformación de la educación superior — masiva y cada vez más minuciosamente diferenciada - que deja de justificarse en nombre de "grandes relatos" o "ideas" fundantes y, en cambio, se hace parte del fenómeno de creciente instrumentalización del conocimiento en todos los ámbitos. En breve, es lo que Lyotard — padre del término tal como aquí lo empleamos - describió como el irresistible avance de la performatividad; esto es, el predominio del "criterio tecnológico" en todos los ámbitos de la cultura; el principio de la más eficiente razón entre insumo y producto; en fin, la mercantilización del conocimiento convertido en commodity. ${ }^{17}$

Fin de los grandes "relatos" o de los discursos sobre la "idea de la universidad" moderna no significa, sin embargo, ausencia de variadas narrativas y lecturas sobre esta institución. Seguramente aquella que hoy domina en nuestro campo de debate sea la narrativa del "capitalismo académico"18, cuyas reglas de economía política y expresión en políticas neoliberales explicarían las transformaciones contemporáneas de la educación superior: diferenciación y masificación de los sistemas nacionales; aparición de nuevos proveedores privados; competencia entre organizaciones académicas por personal, recursos y prestigio; débil coordinación provista por mercados desregulados; obligación para las instituciones -incluidas las universidades estatales - de obtener una parte de sus ingresos mediante el cobro de aranceles y la venta de servicios y productos de conocimiento, y un Estado subsidiario que se limita a financiar la libre elección de oportunidades de estudio (soberanía del consumidor) y a suplir las fallas del mercado.

Como resultado de esas transformaciones se generaría una serie de contradicciones culturales que - se sostiene - acabarían por negar las "ideas" e ideales propios de la tradición de la universidad moderna.

${ }^{17}$ Jean-François Lyotard, The Postmodern Condition: A Report on Knowledge, traducido por Geoff Bennington y Brian Massumi (Manchester: Manchester University Press, 1984). Original: La Condition Postmoderne: rapport sur le savoir (Paris: Minuit, 1979).

${ }^{18}$ Sheila Slaughter y Gary Rhoades, Academic Capitalism and the New Economy: Markets, State, and Higher Education (Baltimore, MD: The Johns Hopkins University Press, 2004). 
Dicho en una frase: la universidad dejaría de ser la sede de la razón y la conciencia más clara de la época para convertirse en un aparato del conocimiento útil; una máquina de desempeños eficientes y rendición de cuentas, donde toda actividad es medida y se ordena en función de la productividad y los beneficios para la economía y la sociedad. La universidad, acusan los críticos, se habría vuelto performativa; es decir, una organización ocupada en asegurar la mejor optimización de todas sus acciones.

III

Frente a este cuadro, el gobierno de la Presidenta Bachelet promete un cambio de paradigma: tanto a nivel programático, sustituyendo un modelo de políticas neoliberales por un modelo socialdemócrata relativamente ortodoxo o nórdico; como también a nivel estructural o de economía política del sistema, pasando de un sistema mixto de provisión coordinado principalmente (se dice) por el mercado a uno gobernado, regulado y financiado principalmente por el Estado. En la práctica, este doble cambio de paradigma debería producir un desplazamiento de la educación superior chilena en tres dimensiones fundamentales: ${ }^{19}$

Primero, desde su actual estatuto de bien de consumo - una mercancía disponible por un precio- a uno que garantice su carácter de derecho social, un bien público de acceso universal gratuito.

Segundo, desde un régimen mixto de provisión conducido y coordinado por el mercado a uno conducido y coordinado más vigorosamente por el Estado, poniéndose fin así al actual déficit de gobierno del sistema y a su crecimiento inorgánico, no-planificado.

$\mathrm{Y}$ tercero, desde un sistema financiado mayoritariamente por recursos privados (de hogares, estudiantes y agentes privados) a uno financiado mayoritariamente por la renta nacional, donde el sector de instituciones estatales (en expansión) operaría en el futuro mediante subsidios directos a los proveedores, mientras las organizaciones privadas, debidamente acreditadas y calificadas por el Estado, se regirían por precios públicos y actuarían bajo estrictas reglas de no-lucro, exigencias de calidad, rendición de cuentas y compromisos de desempeño suscritos con el gobierno.

${ }^{19}$ Véase José Joaquín Brunner, "Un análisis de política de la política educacional”, Debate Universitario 6 (mayo 2005), ISSN en línea 2314 - 1530, 7-15. 
En suma, estaríamos transitando (o buscaríamos hacerlo) desde la concepción de que la universidad moderna deriva su identidad de una "idea" racional fundante a una situación en que diversas narrativas (creencias, ideologías) buscan describir e interpretar el estado de la educación superior e inspirar políticas públicas dirigidas a mantener o a cambiar ese estado de cosas.

Varios elementos pertenecientes a las "ideas" elaboradas a lo largo de la modernidad para dar cuenta de la universidad y fundar su razón de ser son usados ahora difusamente para elaborar estas nuevas narrativas e incidir en el futuro desarrollo del sector.

Las coordenadas básicas en el campo del debate sobre la educación superior son claras. Reducidas a un simple esquema binario, en el ámbito de la economía política los modelos en juego se articulan en torno al Estado o el mercado; en el plano de las políticas públicas, en torno a los paradigmas neoliberal o socialdemócrata.

Las "ideas de universidad" — de Kant a Derrida, del idealismo germano al posmodernismo francés, de ser conciencia de una época a conciencia militante - apenas subsisten como elementos dispersos y descontextualizados dentro de las narrativas que han venido a sustituir a aquellas ideas. A lo más sirven a propósitos polémicos o, en ocasiones, puramente ceremoniales o rituales, cuando no son sólo la expresión de una memoria desolada.

Pudiera ser verdad entonces que la universidad no necesita ya una "razón de ser", una justificación, toda vez que se ha convertido en un aparato central de la educación de masas, la certificación para el trabajo y la producción de conocimiento útil, de la cual se esperan beneficios económicos, de integración social y de difusión cultural.

Si así fuera, si efectivamente hubiese ocurrido que la educación superior se ha convertido en un vital servicio público, una pieza del Estado de bienestar contemporáneo, entonces se entendería que no requiera más una "idea" fundante, sino sólo políticas que aseguren el libre acceso al servicio, la distribución más equitativa posible, procesos y medios efectivos, resultados eficientes, transparencia y rendición de cuentas.

En suma, la universidad posmoderna estaría plenamente instalada y justificada en el reino de la performatividad. De ahora en adelante, las cuestiones que antes se abordaban especulativamente en el terreno 
de las grandes "ideas" se resolverían mediante arreglos pragmáticos del estilo: cuánto Estado, cuánto mercado, y mediante qué instrumentos de política socialdemócrata o neoliberal. El resto queda entregado a consideraciones de productividad y mediciones de eficiencia funcional. EP 